\title{
Unsilencing Suffering: Promoting Maternal Mental Health in Neonatal Intensive Care Units
}

\section{Authors:}

Jessica X. Ouyang, MD, ${ }^{1}$ Jessica L.W. Mayer, $\mathrm{MD}^{2}$, Cynthia L. Battle, Ph.D. ${ }^{3}$, Joanna E. Chambers, $\mathrm{MD}^{4}$, Zeynep N. Inanc Salih, MD, $\mathrm{MA}^{2}$

\author{
Affiliations: \\ ${ }^{1}$ Warren Alpert Medical School of Brown University, Women \& Infants Hospital of \\ Rhode Island, Providence, RI \\ ${ }^{2}$ Indiana University School of Medicine, Riley Hospital for Children, Indianapolis, IN \\ ${ }^{3}$ Warren Alpert Medical School of Brown University, Butler Hospital, Women \& Infants \\ Hospital of Rhode Island, Providence, RI \\ ${ }^{4}$ Department of Psychiatry, Indiana University School of Medicine, Indianapolis, IN
}

Address correspondence to: Jessica X. Ouyang, 101 Dudley Street, Providence, RI 02905,(619) 977-0099, xiaoxi.ouyang@gmail.com

Funding Source: American Academy of Pediatrics Resident Research Grant 2019

Financial Disclosure: The authors have no financial relationships relevant to this article to disclose.

Conflict of Interest: The authors have no conflicts of interest to disclose.

\section{Education Gaps:}

1. Though mothers of infants in the Neonatal Intensive Care Unit (NICU) are at significantly higher risk of poor mental health outcomes, their suffering is often silent - under-recognized and under-treated as the focus is nearly exclusively on the infant(s).

2. Maternal mental health has a direct impact on short-term infant health outcomes while the infant is in the NICU and long-term neurodevelopmental and behavioral outcomes of the infant.

3. There is a lack of standardized screening tools and treatment options to address this vulnerable population, yet new strategies for screening and intervention are being developed.

4. Increased awareness of maternal mental health issues among neonatologists and affiliated NICU staff may lead to expansion of clinical services for mothers of NICU infants, resulting in better outcomes for their offspring.

\section{Learning Objectives:}

1. Become familiar with the maternal mental health care needs in the NICU

2. Recognize how maternal mental health impacts newborn health in the NICU and beyond

This is the author's manuscript of the article published in final edited form as: 
3. Describe the existing interventions for maternal mental health and discuss current gaps and direction for future research
Abbreviation:
NICU - Neonatal Intensive Care Unit
ASD - acute stress disorder
PTSD- post-traumatic stress disorder
CAM - complementary and alternative medicine
NPA- National Perinatal Association 


\begin{abstract}
:
Mothers of infants in the Neonatal Intensive Care Unit (NICU) suffer higher rates of psychological distress, anxiety, and depression compared to the general population. Often, their mental health concerns remain under-identified and under-treated, which can have deleterious effects on the offspring, both in short-term outcomes while in the NICU as well as long-term neurodevelopmental and behavioral outcomes. In this review, we present an overview of existing empirical evidence about how maternal mental health affects the health of their offspring, special considerations regarding the mental health needs of NICU mothers, and the findings about existing and developing interventions to address mental health concerns in this vulnerable population.
\end{abstract}


Postpartum depression is the most common obstetric complication ranging from $13 \%$ to $19 \%$ in the general population (1), with a recent meta-analysis reporting a worldwide average of $17 \%$. (2) Mothers of infants in the Neonatal Intensive Care Unit (NICU) are at increased risk, with a staggering $39 \%$ to $63 \%$ of mothers suffering from postpartum depression and $28 \%$ to $70 \%$ expressing significant psychological distress, anxiety, or trauma-related symptoms. (3)(4)(5)(6) With rates of NICU utilization rising as evidenced by a $22 \%$ increase in NICU utilization in 5 years (approximately $7.8 \%$ of all infants born in the US required NICU services in 2012 compared to $6.4 \%$ in 2007), more mothers are at risk for adverse mental health outcomes. (7)

Compared to maternal mental health research, less is known about paternal mental health in the NICU, though studies have also reported increased levels of distress among NICU fathers. While NICU mothers share some of the same risk factors for postpartum mood and anxiety disorders with the general postpartum population, they have a unique set of additional risk factors related to having a baby in the NICU (see Table).

In addition to depression, maternal mental health studies in the NICU have identified high rates of acute stress disorder (ASD; $23 \%$ to $33 \%$ ) and anxiety disorders $(12.1 \%$ to $29.7 \%) .(6)(12)$ There is a strong link between ASD and later development of post-traumatic stress disorder (PTSD), with a subsequent increase in the risk for depression, overall distress, and vulnerable child syndrome. (12) Unfortunately, in spite of these known rates and risks, mental health conditions among NICU mothers remain largely under-identified and under-treated. (4)

Although multiple professional organizations including the American Academy of Pediatrics (13), National Perinatal Association (14), American College of Obstetrics and 
Gynecology (15), and the US Preventative Services Task Force (16) have put forth recommendations for routine screening for maternal depression (17), universal screening protocols in the NICU to assess for parental distress and mental health adversity currently do not exist. (5) Barriers to screening include lack of knowledge and training among NICU staff to implement screening protocols. Without routine screening in the NICU setting and the dearth of appropriate treatment services available in the community where mothers can be referred to obtain support (17), many mothers with mental health issues suffer in silence - with inadequate support - for extended periods of time. Thus, failure to screen, identify, and treat can lead to devastating consequences for both mother and her infant(s). In order to more readily promote mental health care in the NICU, changes in policy and clinical practices must be evaluated and implemented.

\section{Impact of Maternal Mental Health on Infant Outcomes}

Maternal mental health conditions have a significant direct impact on the offspring, affecting outcomes both in the NICU as well as long-term neurodevelopmental and behavioral health. (18) Increased maternal stress and depressive symptoms may lead to low quality interactions with the infant that can affect early bonding and attachment as well as maternal parenting behavior even five years after NICU discharge. (19) The lack of engagement during mother-child interactions and heightened negativity in depressed mothers is thought to increase risk for insecure attachment, reduced self-regulation, and impaired neurodevelopment in the child, which are known risk factors for childhood and adolescent depression. (8) Alleviating maternal stress can improve the ability of mothers to bond with their infants in the NICU, improve maternal mental health, and ultimately optimize neurodevelopmental and behavioral outcomes of these children. (19) 
When an infant is admitted to the NICU, separation from parents, in particular the mother, disrupts the essential early mother-infant bonding for healthy development. More specifically, poor maternal mental health is associated with less participation in infant care (20), less parental readiness for transition after discharge from the NICU (21), delayed discharge from the NICU (21), and less visitation during the NICU hospitalization (22). This may have an immediate impact on the infant's medical course, as studies have found that an increased maternal presence, namely skin-to-skin contact and hearing of the mother's voice, have led to improved autonomic stability (23), decreased apneic and bradycardic events (24), earlier enteral feeding (25), increased breastfeeding (26), and reduction in medical morbidity including decreased incidence of bronchopulmonary dysplasia, cholestasis, and nosocomial infections (27). Additionally, it is known that the premature brain is more susceptible to environmental stressors in terms of its development and growth. For example, the brain in the third trimester is characterized by rapid growth in a variety of neuronal cell types. (28) There is evidence that an infant's cortisol expression pattern, autonomic and neuroendocrine responses, and gray and white matter maturation can be altered in response to maternal and environmental stress. (29)(30) Cortisol is particularly important during the early months after birth as it has a bipartite effect on the developing brain, having neurotrophic influences at low levels, but becoming neurotoxic when elevated. (31) Chronic levels of high cortisol in the infant, even in the first few months of age, can therefore cause neurotoxic injury to the infant's brain. Oxytocin, a hormone generated through bonding and attachment behaviors in both the mother and the infant, is likely the main inhibitor of cortisol production. (31) In addition to skin-to-skin 
time between infant and mother, positive and sensitive parent interactions may also be protective for premature infants' brain development in the NICU. (30) Animal studies suggest that maternal behaviors toward their newborns may result in epigenetic alterations, leading to changes in downstream thyroid and serotonin signaling. This may later impact emotional and stress regulation throughout the lifespan and into adulthood, either positively or negatively. (29) Hence, interactions that lead to an increase in oxytocin via skin-to-skin contact, breastfeeding, and other positive maternal-child attachment interactions are of primary importance in preventing brain injury in these infants.

Although research examining the impact of compromised maternal mental health on long-term outcomes of NICU infants is still limited, some key findings are emerging. One recent study identified that maternal anxiety during an infant's NICU stay was predictive of worse fine motor skills at 20 months corrected gestational age (28), and was negatively correlated with cognitive and language development. (32) Another longitudinal study found that the infants whose mothers had higher depressive symptoms in the perinatal and first postpartum year had worse behavioral outcomes such as hyperactivity and inattention, physical aggression, emotional and anxiety symptoms, and separation anxiety. (33) Increased maternal stress and depressive symptoms in the NICU were found to be predictive of intrusive and negative parenting behaviors five-years after discharge. (19) Additionally, poor maternal mental health has been correlated with impaired cognitive and physical development of infants, increased utilization of emergency department services, and underutilization of primary preventative services. (14)(33)(34) Finally, unsurprisingly, poor adherence to anticipatory guidance provided by pediatricians such as putting infants 
on their backs to sleep, breastfeeding initiation and maintenance, proper car seat usage, and electric outlet coverage have been described in these parents. (18)(35)

Although less is known about NICU fathers specifically, it has been suggested that depression in fathers of newborns is associated with a number of negative outcomes including greater partner discord, increased maternal depression, higher risk of children demonstrating oppositional behaviors, decreased paternal involvement such as less consistent reading to children, and harsher parenting behaviors such as corporal punishment. (14)(36)

Taken together, it appears that there is a neurobiological vulnerability during the NICU period and that positive mother-child interactions can be protective. However, if this is disrupted by poor maternal mental health conditions, it would not only impact the more immediate medical outcomes in the NICU, but also independently impact the child's development long after discharge from the NICU. It is therefore critically important to screen and develop effective, feasible, and acceptable interventions for mothers. (19)(37)

\section{Interventions Addressing Mental Health among NICU Mothers}

While a number of studies aiming to improve maternal mental health in the NICU have been conducted, a definitive treatment that is feasible, acceptable, and efficacious across NICU settings and populations has not been identified so far. A recent meta-analysis examining interventions in NICU settings that targeted mostly maternal mental health outcomes as measured by various stress, anxiety, trauma, and depressive scales, categorized 46 distinct interventions into four main areas: (1) changing NICU medical care processes, (2) complementary and alternative medicine (CAM), (3) family-centered care, and (4) psychotherapy-driven interventions. (38) Positive overall outcomes were found in 
two categories of studies: CAM studies (e.g., acupuncture, journaling, music, infant massage) and family-centered care (e.g., psychosocial support, education, infant development support). Interventions targeting NICU medical care processes (e.g., those modifying some aspects of care such as utilizing single family versus open bay rooming, or changing visitation practices) revealed mixed results with a few studies showing significant changes in stress and anxiety levels while others showed no effect. (38) Similarly, studies focused on psychotherapy were difficult to assess as a combined group as outcomes differed across the various therapy modalities (which included trauma-focused cognitive behavioral therapy, a crisis intervention targeting anxiety and trauma symptoms, and others). It is noteworthy that psychotherapy interventions may have lower feasibility of implementation due to limited human resources and trained therapists. However, in this particular analysis, it is also important to highlight that the heterogeneity of the interventions studied complicated the overall study results. Another meta-analysis examining various approaches to psychoeducation delivery and cognitive behavioral therapy and its various adaptations on maternal depressive and/or anxiety symptoms showed that no one model was more effective than the others. (39) As such, research to date has investigated a wide range of interventional approaches to address mental health needs of NICU mothers, yet there is not one intervention that is universally regarded as efficacious and feasible.

An emerging intervention that is being examined as a means to promote maternal mental health in the NICU is the utilization of mindfulness. (38)(40)(41) Mindfulness focuses on the present moment in a non-judgmental way, avoiding the distress of the past and the anxiety regarding the future as much as possible, which in turn can help a mother's 
"affect regulation," that is, her ability to constructively modulate her emotional state in the present. (42) In recent decades, mindfulness has become a widely used technique in therapy and counseling to reduce stress and alleviate impact of symptoms across many mental health conditions and settings. There are multiple controlled-trials and meta-analyses supporting mindfulness practice across psychiatric and psychological diagnoses including, but not limited to, depression and anxiety (43), PTSD (44), psychosis (45), and substance use disorders. (46) Additionally, mindfulness requires minimal tools and training, and importantly, has been found to be safe, effective, and acceptable. (47)(48)

To date, three small single-site, single session mindfulness studies done in the NICU setting have shown promising outcomes. (40)(41)(49) The maternal outcome measures in these studies included stress, depression, anxiety, trauma symptoms, sleep quality, maternal attachment, mindfulness skill acquisition, and feasibility and acceptability. Interventions employed varied from patients using audio (mp3) recordings of mindfulness exercises (40), in-person individual training sessions on reading baby cues mindfully (41), and in-person training sessions instructing mindfulness exercises like relaxing sighs throughout NICU stay as well as reviewing calming phrases daily (49). Study outcomes included significant decreases in NICU-related distress scores compared to control groups (41), improvements in post-intervention depressive, anxiety, and trauma symptoms, and improved sleep quality (40). Parents reported high satisfaction and acceptability of these interventions. (40)(41)(49) Additionally, mindfulness studies have shown an association with shorter length of stay independent of gestational age. (41)

Careful considerations ought to be given to how a group-based mindfulness intervention might optimally support all NICU mothers. The single-session individualized 
mindfulness pilot trials suggest that this may be a low-risk, low-cost (48), adaptable intervention that may be acceptable and feasible across a variety of in-person or virtual formats. (39)(40)(41)(49) It is well-established that external social support can help with transitions and coping. In the NICU setting, there are multiple studies showing benefits of peer support (50)(51)(52), culminating in peer-to-peer support recommendations for NICU parents. (53) It has been shown that peer-to-peer support increases perception of social support, acceptance of the situation, and reduces levels of stress, anxiety, and depression. (53) The existing guidelines from the National Perinatal Association (NPA) further emphasize the importance of peer-support as an additional integral component of addressing perinatal mental health and wellness. (37)(53)

In the postpartum period, mindfulness has been shown to increase one's ability for emotional regulation while reducing stress and symptoms of anxiety and depression. (11)(42) This improvement of symptoms occurs through a cognitive shift that promotes an examination of one's emotional experience. (42) Mindfulness has been shown to decrease maternal rigidity, while increasing maternal flexibility and sensitivity. (11)(54) In so doing, the sensitive and adaptive manner in which a mother responds to her infant's cues and her ability to regulate her own affect will help to develop secure attachment and promote appropriate brain development in the infant. (42) Mindfulness therapy has been shown to increase neuronal activity in the left anterior brain regions, an area that is associated with positive affect and compassion and with reductions in anxiety and negative affect. (55) Importantly, the positive neurobiological changes persisted 4 months following an 8-week mindfulness training intervention. (55) Another desirable aspect of mindfulness practice is that it can be adapted to fit individual needs. Furthermore, mindfulness exercises have been 
shown to produce quick relief from distress and anxiety. (49) From a practical standpoint, mindfulness intervention is lauded for its simplicity in facilitation, cost-effectiveness, and flexibility. (48) A group-based mindfulness intervention in the NICU has the potential to be effective across a range of mental health conditions by increasing both internal coping strategies and external support for the parents. Further investigation to incorporate mindfulness and examine its benefits for families in the NICU setting is thus warranted.

It is important to note that certain challenges have limited research on NICU mental health interventions. First, the range of different outcome measures (e.g. stress level, symptoms of depression, trauma, and anxiety) leads to difficulty in comparing the feasibility and efficacy of various interventions. (5)(14) Second, while the existing studies demonstrate acceptability and even effectiveness of many of the interventions, one recurrent concern among many of the published trials was the low participation rates where it was estimated that at least one-third of eligible NICU mothers chose not to participate in the studies. (39) This introduces selection bias and may influence the outcomes of the studies. Various reasons were reported for lack of engagement, and these were often unclear and inconsistent across studies. Factors that may lead to low maternal engagement in these studies include limited length of NICU stay related to either discharge or transfer of infants, feelings of shame and/or stigma, guilt of leaving the infant's bedside, and emotional distress leading to an inability to decide if they would be able to participate. (39) Ultimately, developing a better understanding of these barriers to engagement will lead to the development of more feasible, acceptable, and impactful interventions.

Based on the existing body of research, as well as considerations regarding the nature of the NICU setting and the needs of the patient population, it is important to 
consider other factors in creating an effective intervention approach. Specifically, the following features may optimize feasibility and acceptability of a treatment approach: (1) having an accessible location, such as being onsite in or near the NICU, (2) having an interdisciplinary treatment team with mental health providers in the NICU, (3) scheduling the group sessions at opportune times to maximize participant availability, (4) scheduling duration of treatment (i.e., number of weeks and sessions) to accommodate the variable NICU admission period, and (5) providing virtual or pre-recorded exercises to increase access.

Current guidelines put forth by the NPA advocate for increased services and support to help new parents navigate the stress and burdens of having a child in the NICU. (37)(53) They note that these supports can be difficult to implement pragmatically and to garner successful parental participation. As a result, successful interventions will often require a comprehensive, interdisciplinary team approach to facilitate and encourage parental engagement in NICU family support programming.

\section{Conclusion and Future Directions}

Maternal mental health directly impacts children's health, and this is especially apparent in the perinatal period. Given what is known about the potential long-lasting implications of poor perinatal maternal mental health on a child's neurodevelopmental outcomes, it is imperative that parents are screened and referred for appropriate perinatal mental health services during this critical time. It is essential that neonatologists and pediatricians recognize that the time in the NICU is a particularly critical moment during which screening, intervention, and referral for perinatal mental health services can be implemented. (17)(42) It is still unclear which forms of interventions may be most 
efficacious in promoting parental mental health. Furthermore, strategies are needed to implement these interventions effectively such that they are sustainable, and parents can readily engage. A range of treatment strategies and structures should be evaluated. Based on the existing literature, one particularly promising intervention to improve maternal mental health may be a group-based mindfulness therapy that is designed to promote peerto-peer support. Future studies should address the significant knowledge gaps on how to organize these as well as identifying the ideal time, location, duration, frequency and session content to promote active engagement of parents and ease of access. 


\section{American Board of Pediatrics Neonatal-Perinatal Content Specification:}

- Know the effects of maternal psychiatric disorders and treatment on the fetus and/or newborn infant

- Know the maternal and infant conditions that may interfere with maternal-infant bonding and potential effects on subsequent development 


\section{References:}

1. Gavin NI, Gaynes BN, Lohr KN, Meltzer-Brody S, Gartlehner G ST. Perinatal depression: a systematic review of prevalence and incidence. Obs Gynecol. 2005;106(5):1071-108

2. Shorey S, Chee CYI, Ng ED, Chan YH, Tam WWS, Chong YS. Prevalence and incidence of postpartum depression among healthy mothers: A systematic review and meta-analysis. J Psychiatr Res. 2018;104:235-248

3. Cherry AS, Blucker RT, Thornberry TS, Hetherington C, McCaffree MA, Gillaspy SR. Postpartum depression screening in the Neonatal Intensive Care Unit: Program development, implementation, and lessons learned. J Multidiscip Healthc. 2016;9:59-67

4. Tahirkheli NN, Cherry AS, Tackett AP, McCaffree MA, Gillaspy SR. Postpartum depression on the neonatal intensive care unit: Current perspectives. Int J Womens Health. 2014;6:975-987

5. Vasa R, Eldeirawi K, Kuriakose VG, Nair G J, Newsom C, Bates J. Postpartum depression in mothers of infants in neonatal intensive care unit: Risk factors and management strategies. Am J Perinatol. 2014;31(5):425-433

6. Roque ATF, Lasiuk GC, Radünz V, Hegadoren K. Scoping review of the mental health of parents of infants in the NICU. J Obstet Gynecol Neonatal Nurs. 2017;46(4):576-587

7. Harrison W, Goodman D. Epidemiologic trends in neonatal intensive care, 20072012. JAMA Pediatr. 2015;169(9):855-862 
8. Bogen DL, Fisher SD, Wisner KL. Identifying depression in neonatal intensive care unit parents: then what? J Pediatr. 2016;179(December):13-15

9. Vigod SN, Villegas L, Dennis CL, Ross LE. Prevalence and risk factors for postpartum depression among women with preterm and low-birth-weight infants: A systematic review. BJOG An Int J Obstet Gynaecol. 2010;117(5):540-550

10. Rogers CE, Kidokoro H, Wallendorf M, Inder TE. Identifying mothers of very preterm infants at-risk for postpartum depression and anxiety before discharge. $J$ Perinatol. 2013;33(3):171-176

11. Stotts AL, Villarreal YR, Klawans MR, et al. psychological flexibility and depression in new mothers of medically vulnerable infants: a mediational analysis. Matern Child Health J. 2019;23(6):821-829

12. Shaw RJ, Bernard RS, De Blois T, Ikuta LM, Ginzburg K, Koopman C. The relationship between acute stress disorder and posttraumatic stress disorder in the neonatal intensive care unit. Psychosomatics. 2009;50(2):131-137

13. Earls MF, Yogman MW, Mattson G, Rafferty J. Incorporating recognition and management of perinatal depression into pediatric practice. Pediatrics. 2019;143(1)e20183259

14. Hynan MT, Mounts KO, Vanderbilt DL. Screening parents of high-risk infants for emotional distress: Rationale and recommendations. J Perinatol. 2013;33(10):748753

15. ACOG Committee Opinion Number 757: Screening for perinatal depression. 2018;132(5):e208-212

16. US Preventive Services Task Force. Interventions to Prevent Perinatal Depression: 
US Preventative Services Task Force Recommendation Statement. JAMA. 2019;321(6):580-587

17. Chambers JE, Denne SC. Pediatric Policy Council. Screening for postpartum depression: obligation and opportunity for pediatricians to improve the lives of children. Pediatr Res. 2019;85(7):923-924

18. Paulson JF, Dauber S, Leiferman JA. Individual and combined effects of postpartum depression in mothers and fathers on parenting behavior. Pediatrics. 2006;118(2):659-668

19. Gerstein ED, Njoroge WFM, Paul RA, Smyser CD, Rogers CE. Maternal depression and stress in the neonatal intensive care unit: associations with mother-child interactions at age 5 years. J Am Acad Child Adolesc Psychiatry. 2019;58(3):350-358.e2

20. Gonya J, Nelin LD. Factors associated with maternal visitation and participation in skin-to-skin care in an all referral level IIIc NICU. Acta Paediatr. 2013;102(2):e53-56

21. McGowan EC, Du N, Hawes K, Tucker R, O’Donnell M, Vohr B. Maternal mental health and neonatal intensive care unit discharge readiness in mothers of preterm infants. $J$ Pediatr. 2017;184:68-74

22. Greene MM, Rossman B, Patra K, Kratovil A, Khan S, Meier PP. Maternal psychological distress and visitation to the neonatal intensive care unit. Acta Paediatr. 2015;104(7):e306-313

23. Rand K, Lahav A. Maternal sounds elicit lower heart rate in preterm newborns in the first month of life. Early Hum Dev. 2014;90(10):679-683 
24. Doheny L, Morey JA, Ringer SA, Lahav A. Reduced frequency of apnea and bradycardia episodes caused by exposure to biological maternal sounds. Pediatr Int. 2012;54(2):e1-3

25. Krueger C, Parker L, Chiu SH, Theriaque D. Maternal voice and short-term outcomes in preterm infants. Dev Psychobiol. 2010;52(2):205-212

26. Moore ER, Anderson GC, Bergman N, Dowswell T. Europe PMC Funders Group Early skin-to-skin contact for mothers and their healthy newborn infants. Cochrane Database Syst Rev. 2012;5(5):CD003519

27. Casper C, Sarapuk I, Pavlyshyn H. Regular and prolonged skin-to-skin contact improves short-term outcomes for very preterm infants: A dose-dependent intervention. Arch Pediatr. 2018;25(8):469-475

28. Greene MM, Rossman B, Meier P, Patra K. Elevated maternal anxiety in the NICU predicts worse fine motor outcome in VLBW infants. Early Hum Dev. $2018 ; 116: 33-39$

29. Sanders MR, Hall SL. Trauma-informed care in the newborn intensive care unit: promoting safety, security and connectedness. J Perinatol. 2018;38(1):3-10

30. Vinall J, Miller SP, Synnes AR, Grunau RE. Parent behaviors moderate the relationship between neonatal pain and internalizing behaviors at 18 months corrected age in children born very prematurely. Pain. 2013;154(9):1831-1839

31. Chambers J. The neurobiology of attachment: from infancy to clinical outcomes. Psychodyn Psychiatry. 2017;45(4):542-563

32. Zelkowitz P, Na S, Wang T, Bardin C, Papageorgiou A. Early maternal anxiety predicts cognitive and behavioural outcomes of VLBW children at 24 months 
corrected age. Acta Paediatr. 2011;100(5):700-704

33. Kingston D, Kehler H, Austin MP, et al. Trajectories of maternal depressive symptoms during pregnancy and the first 12 months postpartum and child externalizing and internalizing behavior at three years. PLoS One. 2018;13(4):e0195365

34. Callo-Quinte G, Del-Ponte B, Ruivo ACO, et al. Maternal depression symptoms and use of child health-care services at The Pelotas 2004 Birth Cohort. $J$ Affect Disord. 2019;253:303-307

35. McLearn KT, Minkovitz CS, Strobino DM, Marks E, Hou W. The timing of maternal depressive symptoms and mothers' parenting practices with young children: Implications for pediatric practice. Pediatrics. 2006;118(1)e174-182

36. Ramchandani PG, Psychogiou L, Vlachos H, Iles J, Sethna V, Netsi E, Lodder A. Paternal depression: An examination of its links with father, child and family functioning in the postnatal period. Depress Anxiety. 2011;28(6):471-477

37. Hynan MT, Hall SL. Psychosocial program standards for NICU parents. $J$ Perinatol. 2015;35(S1):S1-S4

38. Sabnis A, Fojo S, Nayak SS, Lopez E, Tarn DM, Zeltzer L. Reducing parental trauma and stress in neonatal intensive care: systematic review and meta-analysis of hospital interventions. J Perinatol. 2019;39(3):375-386

39. Mendelson T, Cluxton-Keller F, Vullo GC, Tandon SD, Noazin S. NICU-based interventions to reduce maternal depressive and anxiety symptoms: A metaanalysis. Pediatrics. 2017;139(3)e20161870

40. Mendelson T, McAfee C, Damian AJ, Brar A, Donohue P, Sibinga E. A 
mindfulness intervention to reduce maternal distress in neonatal intensive care: a mixed methods pilot study. Arch Womens Ment Health. 2018;21(6):791-799

41. Petteys AR, Adoumie D. Mindfulness-based neurodevelopmental care: impact on nicu parent stress and infant length of stay; a randomized controlled pilot study. Adv Neonatal Care. 2018;18(2):E12-E22

42. Khoury B, Lecomte T, Fortin G, et al. Mindfulness-based therapy: a comprehensive meta-analysis. Clin Psychol Rev. 2013;33(6):736-771

43. Boyd JE, Lanius RA, McKinnon MC. Mindfulness-based treatments for posttraumatic stress disorder: a review of the treatment literature and neurobiological evidence. J Psychiatry Neurosci. 2017;42(6):170021

44. Aust J, Bradshaw T. Mindfulness interventions for psychosis: a systematic review of the literature. J Psychiatr Ment Health Nurs. 2017;24(1):69-83

45. Goldberg SB, Tucker RP, Greene PA, et al. Mindfulness-based interventions for psychiatric disorders: A systematic review and meta-analysis. Clin Psychol Rev. 2018;59:52-60

46. Sharma M, Rush SE. Mindfulness-based stress reduction as a stress management intervention for healthy individuals: a systematic review. J Evidence-Based Complement Altern Med. 2014;19(4):271-286

47. Joseph R, Wellings A, Votta G. Mindfulness-Based Strategies: A Cost-Effective Stress Reduction Method for Parents in the NICU. Neonatal Netw. 2019;38(3):135-143

48. Snyder R, Shapiro S, Treleaven D. Attachment Theory and Mindfulness. J Child Fam Stud. 2012;21(5):709-717. doi:10.1007/s10826-011-9522-8 
49. Butcher PR, Kalverboer AF, Minderaa RB, van Doormaal EF ten Wolde Y. Rigidity, sensitivity and quality of attachment: the role of maternal rigidity in the early socio-emotional development of premature infants. Acta Psychiatr Scand Suppl. 1993;375(3):1-38

50. Marshall A, Guillén Ú, MacKley A, Sturtz W. mindfulness training among parents with preterm neonates in the neonatal intensive care unit: a pilot study. $A m J$ Perinatol. 2019;36(14):1514-1520

51. Franck LS, McNulty A, Alderdice F. The perinatal-neonatal care journey for parents of preterm infants: what is working and what can be improved. $J$ Perinat Neonatal Nurs. 2017;31(3):244-255

52. Williams KG, Patel KT, Stausmire JM, Bridges C, Mathis MW, Barkin JL. The neonatal intensive care unit: Environmental stressors and supports. Int $J$ Environ Res Public Health. 2018;15(1):60

53. Preyde M, Ardal F. Effectiveness of a parent "buddy" program for mothers of very preterm infants in a neonatal intensive care unit. CMAJ. 2003;168(8):969-973

54. Hall SL, Ryan DJ, Beatty J, Grubbs L. Recommendations for peer-to-peer support for NICU parents. J Perinatol. 2015;35(S1):S9-S13

55. Davidson RJ, Kabat-Zinn J, Schumacher J, et al. Alterations in brain and immune function produced by mindfulness meditation. Psychosom Med. 2003;65(4):564570 
Table: Risk Factors for Impaired Maternal Mental Health in the Postpartum Period in the NICU

\begin{tabular}{|c|c|c|}
\hline Biological & Psychosocial & $\begin{array}{c}\text { Additional Risks } \\
\text { Conferred by Having an } \\
\text { Infant in the NICU (4) }\end{array}$ \\
\hline 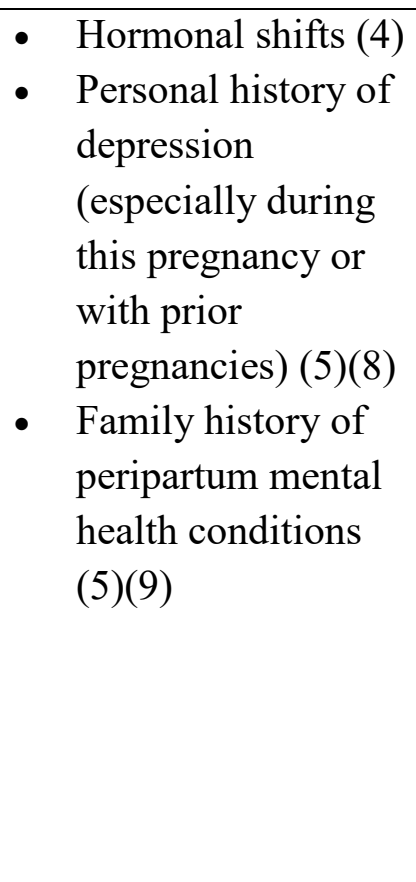 & $\begin{array}{ll}\text { - } & \text { Perceived lack of social } \\
& \text { support/social isolation (4)(9) } \\
\text { - } & \text { Quality of interpersonal } \\
& \text { relationship (especially with } \\
& \text { partner) (4)(5)(10) } \\
\text { - } & \text { Altered parental role (4)(6)(10) } \\
\text { - } & \text { Substance use (5) } \\
\text { - } & \text { Traumatic birth (5)(6) } \\
\text { - } & \text { Younger maternal age (6) } \\
\text { - } & \text { Maternal education (4) } \\
\text { - } & \text { Lower income (4)(6) } \\
\text { - Unmarried mothers (4) (6)(8) } \\
\text { - } & \text { Non-white race (4) } \\
\text { - } & \text { Multiple birth } \\
\text { - } & \text { Psychological inflexibility (11) }\end{array}$ & $\begin{array}{ll}\text { - } & \text { Infant appearance } \\
& (4)(6) \\
\text { - } & \text { Prematurity } \\
\text { (gestational age) (5)(9) } \\
\text { - } \quad \text { Need for ventilatory } \\
\text { support (4)(10) } \\
\text { - } \quad \text { NICU environment } \\
(4)(6) \\
\text { - } \quad \text { Relationship with } \\
\text { NICU team (4)(6) } \\
\text { - Anxiety surrounding } \\
\text { survival and ultimate } \\
\text { health outcomes of the } \\
\text { infant (4) (10) } \\
\text { - Length of stay in the } \\
\text { NICU (4)(5)(6)(10) }\end{array}$ \\
\hline
\end{tabular}

NICU=Neonatal Intensive Care Unit 